\title{
LA ENSEÑANZA DE LA GEOMETRÍA EN LA ESCUELA SECUNDARIA MATERIALES DIDÁCTICOS PARA FAVORECER EL ESTUDIO DE FIGURAS O CUERPOS GEOMÉTRICOS
}

\author{
Alicia Mirta Giarrizzo
}

\begin{abstract}
RESUMEN. Los estudiantes de secundario conocen los conceptos matemáticos de diferentes maneras durante sus trayectorias escolares. Y, si no tienen la posibilidad de enfrentarse a problemas desafiantes a fin de no solo decidir a cuáles de esos conceptos recurrir para resolverlos, sino también remediar los errores que aparecen en sus producciones, es muy probable que puedan tener dificultades para otorgarles sentido. Uno de los errores más frecuentes que se manifiestan en la resolución de problemas geométricos, se vincula con la aplicación de la proporcionalidad directa para calcular perímetros y áreas de figuras geométricas o áreas y volúmenes de cuerpos geométricos cuando varían una o más de sus dimensiones lineales. Considerando lo antes mencionado, con este trabajo se intenta compartir situaciones de enseñanza que fueron propuestas a estudiantes de un tercer año de secundario. Las mismas se basan en la construcción de figuras geométricas y de cuerpos geométricos de diferentes tamaños y formas para favorecer, a partir de su uso como material didáctico, el aprendizaje de contenidos relacionados con la proporcionalidad y la semejanza.
\end{abstract}

\begin{abstract}
Aвstract. High school students knew about the mathematical concepts in different ways during their school trajectory. And, if they do not have the possibility of facing challenging problems in order not only to decide which of those concepts to use to solve them, but also to remedy the mistakes that appear in their productions, it is very likely that they may have difficulty making sense of them. One of the most frequent errors that appear in the resolution of geometric problems is related to the application of direct proportionality to calculate perimeters and areas of geometric figures or areas and volumes of geometric objects when one or more of their linear dimensions vary. Considering the aforementioned, this work tries to share teaching situations that were proposed to high school students. They are based on the construction of geometric figures and geometric shapes of different sizes and forms in order to favor, though their use as didactic material, the learning of contents related to proportionality and similarity.
\end{abstract}

Palabras clave: Geometría, Proporcionalidad, Semejanza, Material didáctico. Keywords: Geometry, Proportionality, Similarity, Didactic material. 
§1. La enseñanza de contenidos geométricos en la educación secundaria

Para Alsina, Burgués, y Fortuny (1988), en la enseñanza de la geometría no se debería:

... sucumbir a las limitaciones formales, simbólicas y algebraicas de los conocimientos matemáticos: será precisamente en este primer estadio de sensibilidad donde el tacto, la vista, el dibujo y la manipulación permitirán familiarizar al alumno con todo un mundo de formas, figuras y movimientos sobre el cual asentar posteriormente los modelos abstractos. (p. 11).

Sin embargo, la utilización de materiales manipulativos como recursos didácticos para la enseñanza de contenidos geométricos va perdiendo presencia en los diseños curriculares o textos para la educación secundaria. Así los estudiantes dejarían atrás ciertos aprendizajes geométricos y no los revisitarían en situaciones problemáticas a fin que reconozcan a la geometría como un medio para describir y modelizar el mundo físico.

Es de esperar que, si durante sus trayectorias escolares se acostumbraron a resolver ejercicios y problemas por medio de la proporcionalidad directa, apliquen sus propiedades para fundamentar las leyes de formación de los perímetros, de las áreas y de los volúmenes de figuras y de cuerpos geométricos —según corresponda- cuando varían una o más de sus dimensiones lineales.

La enseñanza de la geometría debe propiciar entonces avances en el desarrollo de habilidades específicas clasificadas por Bressan, Bogisic, y Crego (2006) en cinco áreas: visuales, de dibujo y construcción, de comunicación, de pensamiento y de aplicación o transferencia. Las habilidades visuales y las habilidades de dibujo y construcción contribuyen durante la etapa de trabajo exploratorio a la identificación de conceptos, relaciones y propiedades geométricas de manera intuitiva para luego avanzar paulatinamente a sus demostraciones según los conocimientos disponibles de los estudiantes. Al respecto, de Guzmán (2010) puntualiza que:

Las ideas, conceptos, y métodos de la matemática presentan una gran riqueza de contenidos visuales, representables intuitivamente, geométricamente, cuya utilización resulta muy provechosa, tanto en las tareas de presentación y manejo de tales conceptos y métodos como en la manipulación con ellos para la resolución de los problemas del campo. (p. 15).

El desafío para los docentes es proponer actividades a sus estudiantes que se resuelvan por medio de representaciones en diferentes marcos con recursos didácticos variados y que además puedan sostenerse en el tiempo para ayudarlos a reconstruir significativamente sus conocimientos. En este sentido, Duval (2006) señala que: 
... la actividad matemática requiere una coordinación interna, que ha de ser construida, entre los diversos sistemas de representación que pueden ser elegidos y usados; sin esta coordinación dos representaciones diferentes significarán dos objetos diferentes, sin ninguna relación entre ambos, incluso si son dos "contextos de representación" diferentes del mismo objeto. (p. 145).

Considerando lo expresado por los autores citados y por otros especialistas que acuerdan con estas concepciones, en este artículo se presentan propuestas didácticas sobre problemas que abordan contenidos relacionados con la semejanza de figuras geométricas y de cuerpos geométricos pretendiendo contribuir a la enseñanza de la noción de proporcionalidad con el propósito de remediar algunos errores persistentes que se observan en los procedimientos, fundamentaciones y/o respuestas de los estudiantes de secundario.

\section{§2. ¿Materiales didácticos o recursos didácticos?}

Alsina y cols. (1988) consideran los materiales como todos aquellos objetos, aparatos o medios de comunicación que pueden ayudar a describir, entender y consolidar conceptos fundamentales en las diversas fases de aprendizaje. Para Hernán y Carrillo (1988) y para Area, Parcerisa, y Rodriguez (2010) los recursos incluyen a los materiales didácticos. En cambio, Coriat (1997) diferencia materiales de recursos, entendiendo por recurso didáctico a cualquier material no diseñado específicamente para el aprendizaje de un concepto o procedimiento que el docente decide incorporar en sus enseñanzas y por material didáctico al que diseña con fines educativos.

González Marí (2010) nombra algunos recursos ${ }^{1}$ que los docentes usan en sus clases: pizarrón, tizas, diversos tipos de papeles, calculadoras, tablets, celulares, computadoras, fotografías, medios audiovisuales y de comunicación, instrumentos de geometría e Internet. $\mathrm{Y}$ también clasifica los materiales didácticos según diferentes criterios. En este trabajo se hará referencia a aquél que distingue los materiales manipulables de los materiales no manipulables o virtuales particularmente diseñados para el aprendizaje de la geometría.

Entre los materiales que se encuentran en el primer grupo están los: policubos, poliminós, bloques, cuerpos geométricos, geoplanos, rompecabezas bidimensionales y tridimensionales, mosaicos y las teselaciones. Y en el segundo grupo se encuentran softwares didácticos (Poly Pro, GeoGebra, Stella 4D, Áreas, Geometer's Sketchpad, etc.), videos educativos (en Youtube, en Canal encuentro, en Educ.ar, etc.) y textos on-line (libros, enciclopedias, revistas, etc.).

\footnotetext{
${ }^{1}$ Se han agregado ejemplos actualizados de otros recursos, materiales manipulables y no manipulables o virtuales.
} 
Los docentes tendrán también que realizar una revisión del uso de los materiales confeccionados por ellos considerando el punto de vista disciplinar para, como se indica en Giarrizzo (2016), “... adecuarlos a los propósitos específicos formulados para cada momento de la secuencia de enseñanza atendiendo la diversidad de conocimientos disponibles de cada grupo de alumnos" (p. 25).

Lo importante, independientemente de cada criterio de clasificación, es que la elección de estos soportes sea plural y esté basada en una evaluación adecuada para que efectivamente favorezcan la comprensión de las diferentes nociones geométricas que inter-juegan y problematizan cada situación.

\section{§3. Situaciones de enseñanza}

Se presenta a continuación una secuencia de actividades que realizaron estudiantes $^{2}$ de tercer año de una escuela secundaria y que integran contenidos de diferentes campos matemáticos para dar respuestas a situaciones intra matemáticas o extra matemáticas interesantes con el propósito de que se involucraran y reflexionaran sobre lo producido formulando conjeturas, estableciendo nuevas relaciones, comunicando modos de resolver, validando sus producciones, planteando nuevos problemas y/o proponiendo proyectos que requieran de las nociones geométricas como herramientas de resolución.

En la presentación de cada una de las actividades, se incluyen algunas de las producciones de los estudiantes con intervenciones de la docente y comentarios que amplían y explican sus vinculaciones con el uso de los materiales didácticos para favorecer sus aprendizajes.

3.1. Primera situación. En la Figura 1 se detallan las actividades propuestas a los estudiantes a fin de diagnosticar sus conocimientos disponibles. Fueron resueltas en cuatro pequeños grupos ${ }^{3}(1,2,3$ y 4). La docente decidió no indicar las medidas iniciales de las dimensiones lineales de las figuras y de los cuerpos geométricos en cada actividad para provocar intercambios durante la puesta en común que propiciaran la argumentación de los procedimientos utilizados y algunos intentos de generalización.

¿Es cierto que dos de las siguientes proposiciones son verdaderas? Dibujen las figuras y los cuerpos geométricos. Realicen los cálculos correspondientes y justifiquen sus respuestas.

a. Si se duplica la longitud de la arista de la base de un prisma y su altura, su volumen también se duplica.

\footnotetext{
${ }^{2}$ La edad promedio de los estudiantes es de 15 años. Todos ellos concurren a una institución escolar de la Provincia de Buenos Aires (Argentina).

${ }^{3}$ Formados por seis integrantes cada uno: A1, A2, A3, .., A24.
} 
b. Al triplicar las longitudes de los lados de un pentágono y las amplitudes de sus ángulos se puede construir otro pentágono semejante.

c. Para que aumente el perímetro de un trapecio isósceles en $5 \mathrm{~cm}$, basta con que aumente en $5 \mathrm{~cm}$ la longitud de su base mayor.

d. Es suficiente que las longitudes de las generatrices, de las alturas y de los radios de las bases de dos conos se reduzcan a la mitad para que la superficie total se reduzca a su cuarta parte.

Figura 1. Actividades de diagnóstico.

Cuando los estudiantes tuvieron que dibujar las figuras y los cuerpos geométricos e iniciar algún procedimiento de resolución formularon preguntas que evidenciaron errores y olvidos: "Profe... ¡se olvidó de poner cuánto miden los lados!", “¿Cuántos lados tiene un prisma?”; “Siempre me confundo, ¿para el volumen va $\mathrm{cm}^{2} \mathrm{o} \mathrm{cm}^{3}$ ?”; “¿Cómo se hacía un pentágono?”; “¿Es ésta la generatriz del cono? (señala la altura)"; “La fórmula que encontré en Internet para calcular el área del círculo nombra el radio, ¿cuál es?" ...

Luego de resolver las actividades, los estudiantes expusieron sus producciones grupales y respondieron las repreguntas y las nuevas preguntas formuladas por la docente para que pudieran validar sus justificaciones y comparar los diferentes procedimientos de resolución. Se comparten algunos de los intercambios que sucedieron entre los alumnos y la docente (D) del curso durante la puesta en común.

D: Vamos ahora a corregir lo que hicieron. Recuerden que lo importante es que expliquen cómo fueron resolviendo las actividades y que no solo compartiremos los procedimientos correctos, sino también los que no terminaron y los que presentan algún tipo de error. Así que espero que todos participen. Comencemos con la actividad a.

A2: Es Verdadera, porque si se duplican las medidas, todo se duplica.

D: ¿Dibujaron los cuerpos geométricos? ¿Alguno de los otros grupos pudo dibujarlos?

A7 pasa a dibujarlos. La docente lo ayuda con la representación tridimensional de los 8 prismas pequeños que están contenidos en el prisma más grande porque tiene dificultades con el trazado de la perspectiva. Les solicita que los representen también usando el GeoGebra para la clase próxima. A22 pide pasar y lo explica de otro modo:

A22: Prisma 1: arista de la base $=2 \mathrm{~cm}$ y altura $=4 \mathrm{~cm}$.

El volumen es: $V=2^{2} \cdot 4=16$. 
Prisma 2: arista de la base $=4 \mathrm{~cm}$ y la altura $=8 \mathrm{~cm}$.

El volumen es: $V=4^{2} \cdot 8=128,8$ veces más.

D: Pero, todos eligieron medidas diferentes. ¿Y llegaron también a que el segundo volumen se obtiene multiplicando por 8 el primer volumen? Vamos a intentar demostrar si se cumple para cualquier medida. Voy a nombrar con la letra a la arista de la base y con la letra $h$ la altura: $V_{1}=a^{2} \cdot h$ y $V_{2}=(2 \cdot a)^{2} \cdot 2 \cdot h$. ¿Cómo podemos escribir $V_{2}$ de una forma equivalente?,

¿quién me va dictando?

A12: Hay que poner: $V_{2}=4 a^{2} \cdot 2 \cdot h$, que después da: $V_{2}=8 a^{2} \cdot h$.

D: ¿Entonces?

A3: ¡Ahí está el 8!

A8: Si se duplica la longitud de la arista de la base de un prisma y su altura, su volumen se tiene que multiplicar por 8.

D: ¿Y si se triplican o se reducen a la mitad las medidas? ¿Y si las bases no son cuadradas? Piénsenlo para mañana... Ahora continuamos con la actividad $b$.

A7: ¡A mí nunca me dan justo los lados del pentágono cuando lo quiero dibujar con el compás!

A21: No dice que es un pentágono regular. Podés dibujar cualquiera.

A1: Pero tenés que medir después los ángulos. Yo lo dibujé regular porque todos miden $72^{\circ}$.

D: ¿Y resultó verdadera o falsa?

A5: Verdadera, porque se mantiene la proporcionalidad.

A1: ¡Falsa! Los ángulos interiores suman en total $1080^{\circ}$. ¿No ves que un ángulo interior es de $108^{\circ}$ y por 3 te da $324^{\circ}$ ?

A5: ¿Y?

A1: ¿Cuánto suman los 5 ángulos interiores si se triplican?

Varios alumnos lo calculan y obtienen $1620^{\circ}$.

D: Si no es un pentágono regular, ¿puede ser otro polígono regular?

A22: Esperen. Tengo que despejar $n$ de la fórmula de los ángulos interiores. ¡Ya está!. Me da 11 lados.

A10: Yo no hice la cuenta. Traté de dibujarlo... Pero no me dan justo los ángulos interiores.

D: ¿Alguno se acuerda cómo hacerlo? 
Como nadie responde la docente les recuerda cómo expresar la amplitud del ángulo interior en grados, minutos y segundos.

\section{$[\ldots]$}

D: Vamos a corregir lo que hicieron para responder la actividad c.

A4: Verdadera, porque si la base menor mide $2 \mathrm{~cm}$, los lados iguales miden $3 \mathrm{~cm} \mathrm{y}$ la base mayor mide $5 \mathrm{~cm}$, el perímetro es de $13 \mathrm{~cm}$. Y si le sumo $5 \mathrm{~cm}$ a la base mayor da $10 \mathrm{~cm}$. Entonces calculamos el perímetro sumando $2 \mathrm{~cm}+3 \mathrm{~cm}+$ $3 \mathrm{~cm}+10 \mathrm{~cm}$ y da $18 \mathrm{~cm}$. O sea, $5 \mathrm{~cm}$ más.

A13: Yo hice varios dibujos y la profe me ayudó a ver qué me faltaba calcular. Y me dio falsa porque el perímetro del segundo trapecio es 21,54 cm y no se obtiene sumándole $5 \mathrm{~cm}$ al del primer trapecio.

D: Contales a tus compañeros cómo lo pensaste y hacé los dibujos en el pizarrón para que lo puedan entender.

En la Figura 2 se muestra lo realizado por A13: primero realiza dos dibujos (1) y (2), pero al agregar los $5 \mathrm{~cm}$ no obtiene un trapecio isósceles. La docente lo orienta para que trace la altura $H$ y para que dibuje aparte los triángulos rectángulos que tienen en común esa altura. Como no distingue los triángulos le propone que sombree uno de ellos (3) y (4). Finalmente, A13 calcula $H$ y $X$ aplicando el Teorema de Pitágoras.

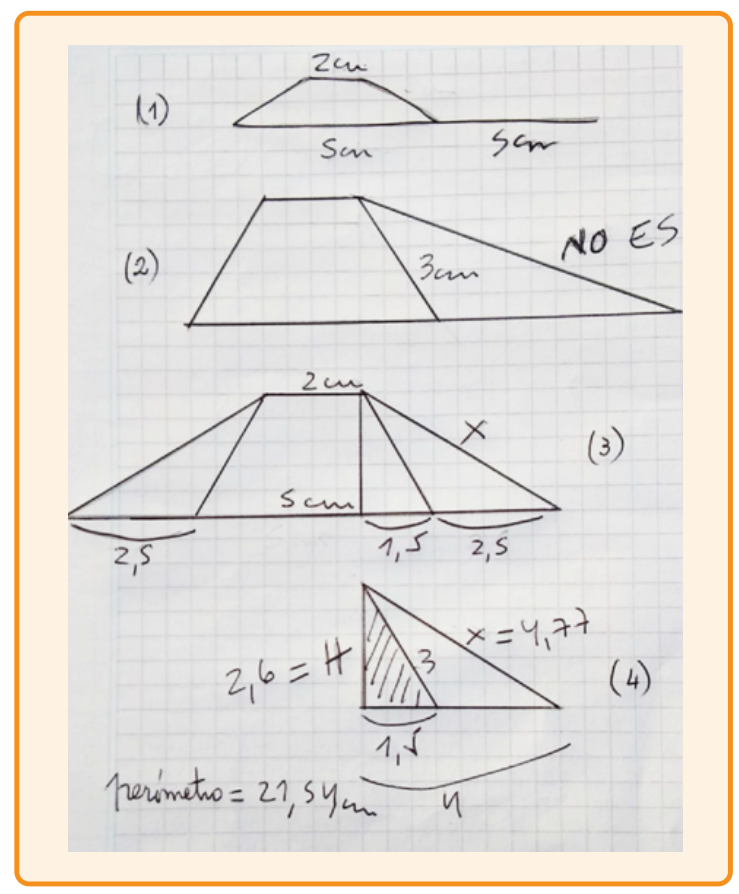

FIgURA 2. Representaciones de A13 para resolver la actividad c de diagnóstico. 
D: ¿Alguna pregunta?

La docente aclara las dudas de algunos alumnos.
$[\ldots]$
D: ¿Corregimos la actividad d?

A17: Falsa, porque se tiene que también reducir a la mitad. Es una regla de tres.

A24: A mí me dio verdadera.

D: ¿Cómo lo justificaste?

A24: No los dibujé... Lo pensé con las fórmulas...

D: Vamos a ver tu resolución.

A24 pasa al pizarrón y escribe:

Primer cono:

$$
A T=\pi r(r+g)=3,14 \cdot 3 \cdot(3+5)=75,36 ;
$$

Segundo cono:

$$
A T=\pi r(r+g)=3,14 \cdot 1,5 \cdot(1,5+2,5)=18,9 ;
$$

y $75,36=18,9 \cdot 4$.

Si las longitudes de las generatrices, de las alturas y de los radios de las bases de dos conos se reducen a la mitad, la superficie total se reduce a su cuarta parte.

Entre las dificultades transversales y más comunes en las resoluciones de todos los casos planteados se diagnosticó la imposibilidad, por parte de los estudiantes, de procesar imágenes mentales que les permitieran anticipar si las transformaciones geométricas propuestas eran verdaderas o falsas, dibujar cuerpos geométricos en perspectiva, indicar unidades de medida y usar diferentes instrumentos de geometría.

Durante los intercambios producidos en la puesta en común reconocieron sus errores y tomaron apuntes sobre las aclaraciones dadas por la docente y los aportes de sus compañeros. Resultó fundamental la confianza dada por la docente al habilitar la participación de todos los alumnos independientemente de las actividades que lograron resolver.

3.2. Segunda situación. Se mantuvieron los mismos integrantes de los cuatro grupos de trabajo que participaron en la primera situación. La docente decidió no indicar los recursos para darles a los estudiantes la posibilidad de elegirlos. 
En actividades anteriores ya habían representado todos los hexaminós ${ }^{4}$ que pueden formarse $y$, de las 35 posibilidades, reconocieron las 11 que se usan como desarrollos planos para construir un cubo. El resto de los desarrollos planos, que necesitaron para construir los cuerpos geométricos para resolver las actividades de esta situación (Figura 3), los buscaron en diferentes libros y en algunos sitios de Internet ${ }^{5}$.

1. Construyan un prisma de base hexagonal, una pirámide de base hexagonal, un cubo y un prisma de base cuadrada. Elijan las longitudes de las dimensiones lineales de modo que:

a. el volumen del prisma de base hexagonal sea el triple del volumen de la pirámide de base hexagonal.

b. el área total del cubo sea la cuarta parte del área total del prisma de base cuadrada.

2. Construyan tres cuerpos geométricos cuyas dimensiones lineales respondan a alguna ley de formación y establezcan relaciones entre sus áreas laterales, sus áreas totales y sus volúmenes. Enuncien conclusiones.

Figura 3. Actividades para relacionar las áreas y los volúmenes de cuerpos geométricos.

\section{Actividad 1.a.}

Grupo 1. Sus integrantes buscaron información en Internet y encontraron actividades mediadas por el software GeoGebra. Copiaron las respuestas y concluyeron que la relación era falsa: "el volumen del prisma de base hexagonal no es el triple del volumen de la pirámide de base hexagonal si tienen bases y alturas iguales".

En las Figuras 4 y 5 se observan los sectores de las actividades que los estudiantes consultaron para calcular los volúmenes del prisma y de la pirámide usando GeoGebra. Al confrontar estas respuestas con las de los otros grupos surgió la necesidad de realizar los cálculos completos para corregirlos:

$$
14,24 \cdot 3=42,72 \neq 51,96
$$

$\mathrm{y}$

$$
51,96: 3=17,32 \neq 14,24
$$

Reconocieron que las aproximaciones influyeron en los resultados que copiaron. La docente intervino para que calcularan los volúmenes reemplazando la apotema de la base por $\sqrt{3}$ y así pudieron establecer que la relación entre los volúmenes de

\footnotetext{
${ }^{4}$ Un hexaminó está formado por seis cuadrados unidos por los lados, de tal forma que cada dos de ellos tienen al menos un lado en común.

${ }^{5}$ Luego de intercambiar algunas direcciones acordaron consultar: Matemáticas visuales. Prismas y sus desarrollos planos en http: / / www . matematicasvisuales.com/html/geometria/ planenets/prismas.html
} 
la pirámide $V_{1}$ y del prisma $V_{2}$ era verdadera:

$$
V_{1}=\frac{\frac{P \cdot a p}{2} \cdot h}{3}=\frac{\frac{12 \cdot \sqrt{3}}{2} \cdot 5}{3}=10 \sqrt{3}
$$

$\mathrm{y}$

$$
V_{2}=\frac{P \cdot a p}{2} \cdot h=\frac{12 \cdot \sqrt{3}}{2} \cdot 5=30 \sqrt{3}
$$
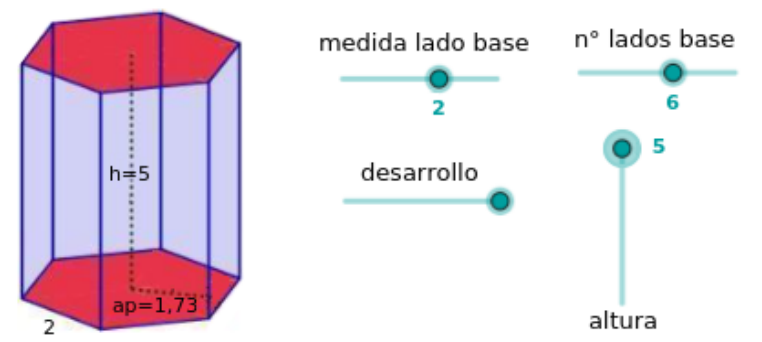

$$
\begin{aligned}
& V \text { Volumen } \\
& V=\text { Área base } x \text { altura } \\
& V=\frac{\text { Perímetro } \times \text { ap }}{2} \times \mathrm{h} \\
& V=\frac{6 \times 2 \times 1.732}{2} \times 5 \\
& V=51.96 \boldsymbol{u}^{8}
\end{aligned}
$$

FIGURA 4. Volumen del prisma hexagonal usando GeoGebra ${ }^{6}$.
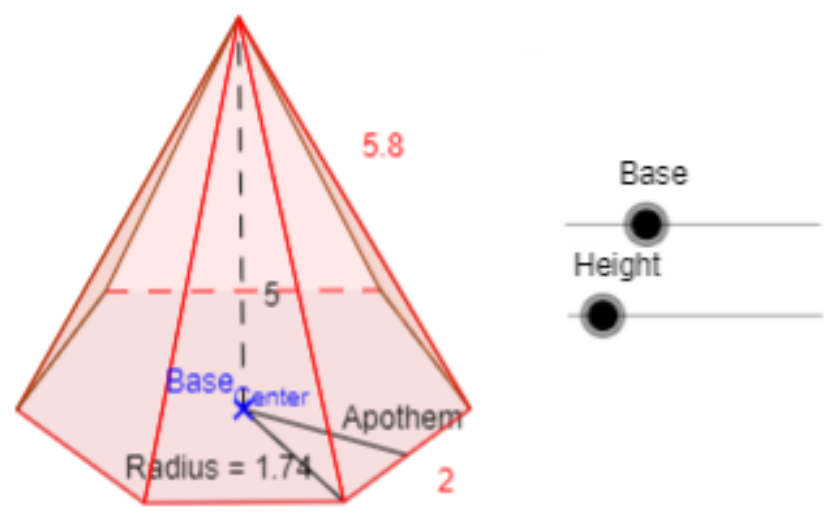

Solution

Apothem $=1.42$

Base area $=8.55$

One lateral side area $=5.72$

Lateral surface $=34.3$

Total surface $=42.85$

Volume $=14.24$

Figura 5. Volumen de la pirámide hexagonal usando GeoGebra ${ }^{7}$.

\section{Actividad 1.b.}

Grupo 3. Los estudiantes consideraron un cubo de $2 \mathrm{~cm}$ de arista y un prisma en el que la arista de la base también era de $2 \mathrm{~cm}$ y la altura de $8 \mathrm{~cm}$ porque pensaron que tenían que cuadruplicarla para que el área total se cuadruplicara. Obtuvieron que el área total del cubo era de $24 \mathrm{~cm}^{2}$ y que el área total del prisma de base cuadrada era de $72 \mathrm{~cm}^{2}$. Entonces, con estas medidas llegaron a enunciar que: "el área total del cubo es la tercera parte del área total del prisma de base cuadrada".

La docente les propuso cuadricular las bases y las caras laterales de esos cuerpos tomando como unidad $1 \mathrm{~cm}$ y que contaran los cuadrados de $1 \mathrm{~cm}^{2}$ de área que se

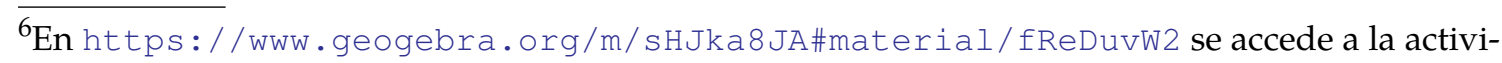
dad completa.

${ }^{7}$ En https://www geogebra.org/m/na69S9vm se accede a la actividad completa. 


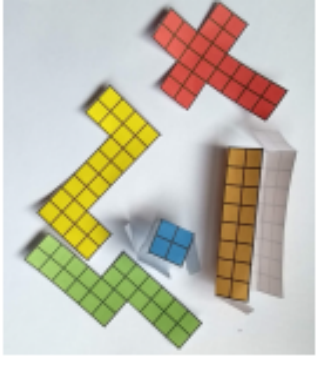

Desarrollos planos

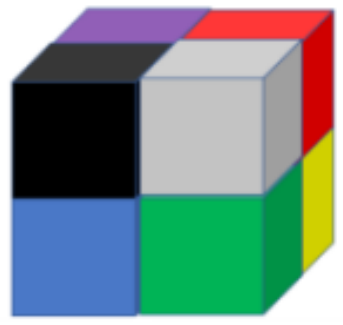

Paint

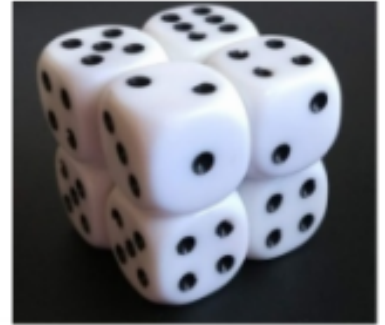

Dados convencionales

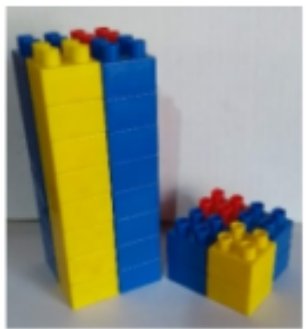

Ladrillos encastrables

FIgURA 6. Materiales utilizados por los estudiantes para representar diferentes situaciones.

habían formado. Otros grupos eligieron diferentes materiales como soportes para resolver esta actividad (Figura 6).

El siguiente intercambio entre estudiantes da cuenta de cómo el uso de estos materiales favoreció la interpretación de las unidades de longitud, de superficie y de volumen.

A13: ¡En el cubo son 24 cuadraditos y en el prisma son 72 !

A14: ¿Entonces en el otro problema tenemos que pensar que son cubitos de $1 \mathrm{~cm}$ de arista? ¡Hay que imaginarse que los llenamos con agua y los vamos poniendo adentro! Y ahí tenemos el volumen...

A15: ¡Sí! Pero también podemos imaginarnos el cubo mágico que está formado por 27 cubitos de $1 \mathrm{~cm}$ de arista. Si cada cubito tiene $1 \mathrm{~cm}^{3}$ de volumen, contándolos sacamos el volumen del cubo grande.

A16: Se ve que en cada cara hay 9 cuadraditos de $1 \mathrm{~cm}^{2}$. Y si sumamos todos los cuadraditos de las seis caras hay 54, que es el área total del cubo grande. Por eso se pone "centímetros cuadrados". Porque son cuadrados de $1 \mathrm{~cm}$ de lado.

A14: En el volumen va "centímetros cúbicos" porque son cubitos de $1 \mathrm{~cm}$ de arista.

A13: ¡Ahora entendí la diferencia!

Grupo 2. Calcularon el área total de un cubo de $2 \mathrm{~cm}$ de arista: $24 \mathrm{~cm}^{2}$. La cuadruplicaron y plantearon la siguiente ecuación: $96=8+8 x$. Luego enunciaron: "para que el área total del cubo de $2 \mathrm{~cm}$ de arista sea la cuarta parte del área total del prisma de base cuadrada, la base tiene que ser la misma y la altura tiene que medir $11 \mathrm{~cm}$ ". La docente les propuso que, con el mismo procedimiento, consideraran que la arista de la base aumentara sucesivamente en $1 \mathrm{~cm}$ para encontrar, si es posible, una ley de formación.

Grupo 4. Sus integrantes comenzaron calculando las áreas laterales y totales de los prismas de base cuadrada manteniendo la longitud de la arista de la base constante de $3 \mathrm{~cm}$ y sus alturas de $3 \mathrm{~cm}, 6 \mathrm{~cm}, 9 \mathrm{~cm}$ y $12 \mathrm{~cm}$. Como la docente observó que 
los cálculos estaban desordenados, les sugirió que tabularan las alturas, las áreas laterales y las áreas totales de los prismas (Tabla 1).

\begin{tabular}{|c|c|c|c|}
\hline Área de la base & Altura & Área lateral & Área total \\
\hline $9 \mathrm{~cm}^{2}$ & $3 \mathrm{~cm}$ & $36 \mathrm{~cm}^{2}$ & $54 \mathrm{~cm}^{2}$ \\
\hline $9 \mathrm{~cm}^{2}$ & $6 \mathrm{~cm}$ & $72 \mathrm{~cm}^{2}$ & $90 \mathrm{~cm}^{2}$ \\
\hline $9 \mathrm{~cm}^{2}$ & $9 \mathrm{~cm}$ & $108 \mathrm{~cm}^{2}$ & $126 \mathrm{~cm}^{2}$ \\
\hline $9 \mathrm{~cm}^{2}$ & $12 \mathrm{~cm}$ & $144 \mathrm{~cm}^{2}$ & $162 \mathrm{~cm}^{2}$ \\
\hline
\end{tabular}

TABla 1. Áreas laterales y totales de los prismas (Grupo 4).

Observaron y enunciaron que la ley de formación de las áreas laterales es la misma que la ley de formación de las alturas: "si se multiplica la altura del primer prisma por dos, por tres y por cuatro pasa lo mismo con las áreas laterales". El siguiente diálogo para hallar la ley de formación entre las áreas totales de los prismas tuvo lugar entre los estudiantes y la docente:

D: Si no se obtienen las áreas totales al multiplicar los $54 \mathrm{~cm}^{2}$ por 2, por 3 y por 4, ¿será posible que se obtengan al multiplicarlas por otros números?

A19: Puede ser, pero no me doy cuenta para calcularlo.

A20: Vamos probando hasta que dé.

A21: ¿Y 54 por qué número nos da 90?

D: Ese tipo de pregunta, ¿a qué les recuerda?

A22: ¿Ecuaciones?

A19: (Escribe en su cuaderno.) $54 \cdot x=90 \Longrightarrow x=90: 54=5 / 3$. ¡Pero es una fracción!

D: ¿No es un número?

A20 resolvió las otras ecuaciones y expresó las áreas totales según se muestra en la Tabla 2.

\begin{tabular}{|c|}
\hline Área total \\
\hline $54 \mathrm{~cm}^{2}=54 \mathrm{~cm}^{2} \cdot 3 / 3$ \\
\hline $90 \mathrm{~cm}^{2}=54 \mathrm{~cm}^{2} \cdot 5 / 3$ \\
\hline $126 \mathrm{~cm}^{2}=54 \mathrm{~cm}^{2} \cdot 7 / 3$ \\
\hline $162 \mathrm{~cm}^{2}=54 \mathrm{~cm}^{2} \cdot 9 / 3$ \\
\hline
\end{tabular}

TABLA 2. Relación entre las áreas totales de los prismas según A20. 
A partir de las intervenciones realizadas por la docente pudieron generalizar la relación entre las áreas totales de los prismas con la siguiente fórmula:

$$
A T_{n}=54 \cdot \frac{2 n+1}{3} \text {. }
$$

\section{Actividad 2.}

Grupos 1 y 3. Los integrantes de ambos grupos eligieron tres prismas de base cuadrada siendo la arista de la base de $1 \mathrm{~cm}$ y las alturas de $1 \mathrm{~cm}, 2 \mathrm{~cm}$ y $3 \mathrm{~cm}$. Utilizaron diferentes recursos (Figura 7).

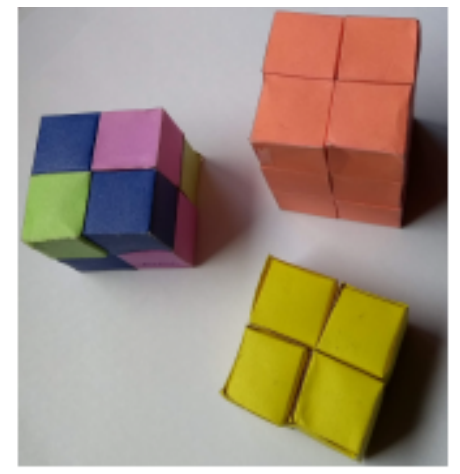

Cubitos en cartulina de $1 \mathrm{~cm}$ de arista

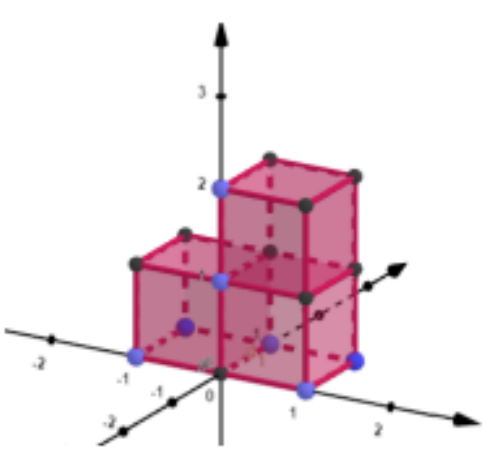

GeoGebra 3D

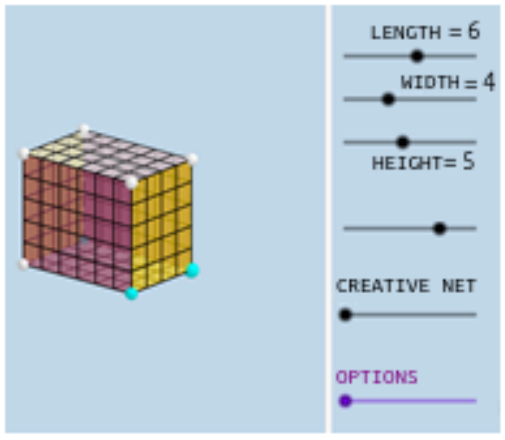

Surface Area: Intuitive Introduction. ${ }^{8}$

FIgURA 7. Representaciones de prismas de base cuadrada.

Calcularon sus áreas laterales, sus áreas totales y sus volúmenes. Los dos grupos llegaron a las mismas conclusiones: "la ley de formación de las áreas laterales y de los volúmenes es la misma que la ley de formación de las alturas" y "la ley de formación de las áreas totales es: $A T_{n}=16 \cdot \frac{n+2}{2}$ ". Considerando el trabajo de los estudiantes cabe indicar que:

Para el estudio de las magnitudes espaciales como el perímetro, el área y el volumen no es suficiente con reemplazar los datos en las fórmulas correspondientes para calcular determinadas incógnitas. Las construcciones con diferentes materiales de los objetos considerados será un recurso fundamental para la comprensión y para la integración de los contenidos mediante la visualización de las modificaciones producidas en ellos durante toda la resolución del problema. (Giarrizzo, 2007, p. 66)

\footnotetext{
${ }^{8}$ GeoGebra Resources. Con los deslizadores se pueden variar las dimensiones lineales de los prismas, la visualización interna de las unidades de área, los desarrollos planos, las caras laterales y las bases.
} 
3.3. Tercera situación. La docente organiza nuevamente los grupos de modo que estén formados por estudiantes que hayan logrado diferentes avances en sus aprendizajes para que compartan sus producciones anteriores con otros compañeros. En la Figura 8 se detallan las actividades correspondientes a esta situación.

En las Tablas 3 y 4 se muestran algunos datos correspondientes a las figuras geométricas y a los cuerpos geométricos que forman series de tres elementos.

a. ¿Cuáles son las leyes de formación de cada serie? Si se agregaran tres nuevas figuras geométricas y tres nuevos cuerpos geométricos a cada una de las series anteriores, ¿qué medidas les corresponderían a sus elementos? Expresen verbalmente y simbólicamente, de ser posible, las leyes de formación encontradas.

b. ¿Se cumplirán las mismas leyes de formación para los perímetros y las áreas de las figuras geométricas que forman cada serie? ¿Y para las áreas totales y los volúmenes de los cuerpos geométricos que forman cada serie? ¿Por qué?

c. ¿Encontraron otras relaciones? Exprésenlas verbalmente y simbólicamente, de ser posible.

Figura 8. Actividades para definir las leyes de formación de diferentes series de figuras y de cuerpos geométricos.

\begin{tabular}{|c|c|c|c|c|c|c|}
\hline & \multicolumn{3}{|c|}{ Rombos } & \multicolumn{3}{|c|}{ Pentágonos regulares } \\
\hline Diagonal mayor & $8 \mathrm{~cm}$ & $16 \mathrm{~cm}$ & $24 \mathrm{~cm}$ & \multicolumn{3}{|c|}{} \\
\hline $\begin{array}{c}\text { Ángulo que determina la diagonal } \\
\text { mayor con el lado }\end{array}$ & $30^{\circ}$ & $45^{\circ}$ & $60^{\circ}$ & & & \\
\hline Lado & & & & & & \\
\hline Perímetro & & & & & & \\
\hline Área & & & & & & \\
\hline
\end{tabular}

Tabla 3. Datos de las figuras geométricas de las series.

\begin{tabular}{|c|c|c|c|c|c|c|c|c|c|}
\hline & \multicolumn{3}{|c|}{$\begin{array}{c}\text { Pirámides de base } \\
\text { hexagonal }\end{array}$} & \multicolumn{3}{|c|}{ Prismas de base cuadrada } & \multicolumn{3}{|c|}{ Conos } \\
\hline Arista de la base & $4 \mathrm{~cm}$ & $4 \mathrm{~cm}$ & $4 \mathrm{~cm}$ & $4 \mathrm{~cm}$ & $8 \mathrm{~cm}$ & $12 \mathrm{~cm}$ & & & \\
\hline Altura & $5 \mathrm{~cm}$ & $10 \mathrm{~cm}$ & $15 \mathrm{~cm}$ & $10 \mathrm{~cm}$ & $20 \mathrm{~cm}$ & $30 \mathrm{~cm}$ & $6 \mathrm{~cm}$ & $10 \mathrm{~cm}$ & $14 \mathrm{~cm}$ \\
\hline Radio de la base & & & & & & & $5 \mathrm{~cm}$ & $5 \mathrm{~cm}$ & $5 \mathrm{~cm}$ \\
\hline Área total & & & & & & & & & \\
\hline Volumen & & & & & & & & & \\
\hline
\end{tabular}

Tabla 4. Datos de los cuerpos geométricos de las series.

Grupo 1. Encontraron dos leyes de formación para los rombos: "las diagonales mayores de los rombos se forman sumándole $8 \mathrm{~cm}$ a la diagonal anterior" $\mathrm{y}$ "las diagonales mayores de los rombos se forman duplicando y triplicando la diagonal mayor del primer rombo".

Después de varios intentos para dibujar los rombos trazando las diagonales mayores y los ángulos que éstas determinaban con el lado solicitaron la ayuda de la docente: 
A1: ¿Cómo sabemos dónde terminan los lados?

D: Piensen en las propiedades de los cuadriláteros.

A2: Las diagonales son perpendiculares y se cortan en su punto medio. ¡Es eso!

Al dibujar los rombos, reconocieron que con los datos del segundo rombo quedaba dibujado un cuadrado y con los datos del tercer rombo la diagonal mayor ya no lo era. La docente dedicó unos minutos para aclarar sus dudas. Luego midieron con la regla los lados y las diagonales menores para determinar sus longitudes.

D: Busquen otra forma de calcularlo. ¿Recuerdan el tema anterior que vimos? Resolvieron problemas parecidos.

A4: Seno, coseno...

D: ¿Con cuál o cuáles de esas funciones pueden calcular los lados de los rombos y las diagonales menores?

A3: (Busca en su cuaderno.) ¡Listo! Con el seno y la tangente de los ángulos que nos diste en la tabla.

Grupo 3. Para la serie de las pirámides de base hexagonal aseguraron que como la base era constante y las alturas respondían a múltiplos de 5, cada volumen también aumentaría 5 veces más que el volumen anterior como se evidencia en la siguiente interacción:

D: Intenten expresar los volúmenes con números irracionales.

A14: ¿Por qué?

D: Porque me parece que se pueden encontrar algunas relaciones...

A15: A ver si me acuerdo... (Los volúmenes están registrados en la Tabla 5)

A13: Por eso no podíamos encontrar la relación, profe. ¿Conviene siempre dejar las raíces sin calcular?

D: Depende de cada situación. En este caso, si se calcula la $\sqrt{3}$, ¿Cómo decidimos cuántos decimales considerar?

\begin{tabular}{|c|c|c|c|}
\hline Arista de la base & $4 \mathrm{~cm}$ & $4 \mathrm{~cm}$ & $4 \mathrm{~cm}$ \\
\hline Altura & $5 \mathrm{~cm}$ & $10 \mathrm{~cm}$ & $15 \mathrm{~cm}$ \\
\hline Volumen & $40 \sqrt{3} \mathrm{~cm}^{3}$ & $80 \sqrt{3} \mathrm{~cm}^{3}$ & $120 \sqrt{3} \mathrm{~cm}^{3}$ \\
\hline
\end{tabular}

TAвla 5. Volúmenes de las pirámides de base hexagonal (Grupo 3). 
Se dieron cuenta que la ley de formación de los volúmenes era la misma que la ley de formación de las alturas:

$$
V_{2}=2 V_{1}, \quad V_{3}=3 V_{1}, \quad h_{2}=2 h_{1}, \quad h_{3}=3 h_{1} .
$$

Decidieron comprobar estas relaciones construyendo las pirámides. Se encontraron con el desafío del armado de sus desarrollos planos y propusieron dos procedimientos: calcular las apotemas laterales a partir de la apotema de la base y de las alturas de las pirámides o, calcular los lados congruentes de las caras triangulares de las pirámides a partir del radio de la base y de las alturas de las pirámides.

Grupo 4. Registraron la variación de las áreas totales de los prismas de base cuadrada (Tabla 6).

\begin{tabular}{|c|c|c|c|c|c|c|}
\hline Arista de la base & $4 \mathrm{~cm}$ & $8 \mathrm{~cm}$ & $12 \mathrm{~cm}$ & $16 \mathrm{~cm}$ & $20 \mathrm{~cm}$ & $24 \mathrm{~cm}$ \\
\hline Altura & $10 \mathrm{~cm}$ & $20 \mathrm{~cm}$ & $30 \mathrm{~cm}$ & $40 \mathrm{~cm}$ & $50 \mathrm{~cm}$ & $60 \mathrm{~cm}$ \\
\hline Área total & $192 \mathrm{~cm}^{2}$ & $768 \mathrm{~cm}^{2}$ & $1728 \mathrm{~cm}^{2}$ & $3072 \mathrm{~cm}^{2}$ & $4800 \mathrm{~cm}^{2}$ & $6912 \mathrm{~cm}^{2}$ \\
\hline
\end{tabular}

TABla 6. Áreas totales de los prismas de base cuadrada (Grupo 4).

Fundamentaron la no existencia de proporcionalidad directa con los cocientes que obtuvieron entre las áreas totales y las aristas de las bases, expresados en $\mathrm{cm}$ : $48,96,144,192,240$ y 288. Pero descubrieron que las diferencias entre esos cocientes eran constantes e iguales a $48 \mathrm{~cm}$. Después pensaron en calcular las diferencias entre las áreas totales. Obtuvieron los siguientes valores expresados en $\mathrm{cm}^{2}: 576$, 960,1344 . 1728, 2112. Y al intentar con la segunda diferencia, encontraron una constante igual a $384 \mathrm{~cm}^{2}$. Decidieron representar gráficamente la relación (Figura 9) ${ }^{9}$ y reconocieron que su comportamiento correspondía a una función cuadrática.

Con ayuda de la docente enunciaron: "si la segunda diferencia entre las imágenes de los valores sucesivos del dominio de una función es constante, la función es cuadrática". Lograron escribir la fórmula de la función que expresa la variación de las áreas totales de los prismas en función de la arista de la base: $A T=12 \cdot a^{2}$. Calcularon los volúmenes de los prismas y escribieron la fórmula de la función que expresa la variación de los volúmenes de los prismas en función de la arista de la base: $V=2,5 \cdot a^{3}$.

Calcularon los cocientes sucesivos entre las longitudes de las aristas de las bases de los prismas y obtuvieron esta sucesión particular $S_{1}: 2 / 1,3 / 2,4 / 3,5 / 4,6 / 4$.

\footnotetext{
${ }^{9}$ Se ha trazado la línea que une las coordenadas que representan gráficamente la situación para que los estudiantes visualicen el comportamiento de la función cuadrática que en este caso es discontinua.
} 


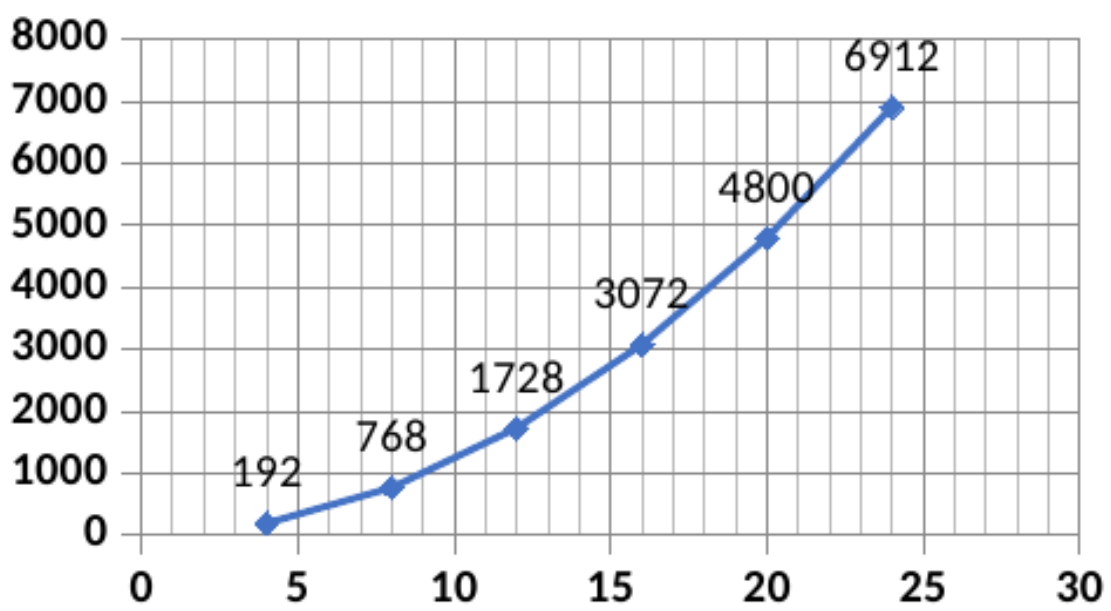

Figura 9. Áreas totales de los prismas en función de la arista de la base (Grupo 4).

Continuaron con los cocientes sucesivos entre las áreas totales de los prismas y obtuvieron la sucesión que se forma con los cuadrados de los números de la sucesión anterior, $S_{2}: 4 / 1,9 / 4,16 / 9,25 / 16,36 / 25$. Finalmente, los cocientes entre los volúmenes de los prismas forman una sucesión cuyos términos son los cubos de los números de la primera sucesión $S_{3}: 8 / 1$, 27/8, 64/27, 125/64, 216/125. Escribieron las fórmulas de sus términos generales y estudiaron analíticamente los comportamientos de sus representaciones gráficas.

$$
\text { Para } S_{1}: a_{n}=\frac{n+1}{2}, \quad \text { Para } S_{2}: a_{n}=\frac{(n+1)^{2}}{2}, \quad \text { Para } S_{3}: a_{n}=\frac{(n+1)^{3}}{2} .
$$

Cabe señalar que no siempre los estudiantes indicaron las unidades de medida correspondientes a cada magnitud y en consecuencia enunciaron equivalencias falsas al comparar solamente las medidas entre las áreas y los volúmenes.

\section{§4. Sobre la evaluación y los proyectos. Una forma diferente de evaluar}

Por cuestiones de espacio se indican algunas orientaciones sobre la evaluación que fue realizada por los estudiantes en dos momentos. Primero se apeló a una actividad que podían resolver en sus casas de modo individual consultando lo registrado en sus carpetas, otros textos y/o sitios de Internet. Para justificar sus respuestas, los ejemplos o contraejemplos debían ser diferentes a los dados en las actividades grupales. Se realizaron tutorías virtuales y se destinaron además las dos clases siguientes a la corrección de la actividad entregada.

A posteriori se realizó una evaluación en la escuela que fue resuelta en parejas formadas por la docente cuyos integrantes habían alcanzado indicadores de avance similares en sus aprendizajes. En las actividades se les proponía inventar, representar y analizar las leyes de formación de dos nuevas series: una formada 
por 5 polígonos regulares semejantes y otra formada por 5 cuerpos geométricos semejantes.

Los proyectos ${ }^{10}$ pusieron en juego los saberes y habilidades logrados por los estudiantes durante la resolución de las actividades propuestas por sus docentes ${ }^{11}$ y se basaron en la construcción de figuras y cuerpos geométricos de diferentes tamaños con igual o diferente forma para que los alumnos de jardines de infantes y escuelas primarias pudieran usarlos como recursos didácticos.

\section{§5. Para finalizar...}

La inclusión de la construcción de figuras y de cuerpos geométricos en la planificación de las situaciones propuestas favoreció la enseñanza y el aprendizaje de los conceptos geométricos a través de su manipulación, observación o lectura ofreciéndoles oportunidades de aprender algo, o bien de intervenir en el desarrollo de alguna función de la enseñanza (Gimeno Sacristán, 2001).

Las producciones de los estudiantes, las intervenciones de la docente, los materiales y recursos didácticos y las orientaciones didácticas que complementaron las actividades seleccionadas pretendieron mostrar otros alcances que las relaciones de la proporcionalidad y semejanza tienen con los contenidos de los distintos campos matemáticos que figuran en los diseños curriculares para la enseñanza de la matemática en la escuela secundaria.

Es importante que los docentes instalen diferentes condiciones didácticas durante la gestión de las clases para que los estudiantes logren avanzar en sus aprendizajes, dándoles la oportunidad de que decidan entre los procedimientos posibles de resolución y los recursos didácticos — provisorios y mejorables-cuál o cuáles son los más adecuados para resolver cada problema geométrico. De este modo se podrán evaluar los procesos de adquisición de los conocimientos con preguntas, repreguntas, comentarios y sugerencias —orales o escritas- para establecer si verdaderamente las resoluciones erróneas o incompletas se producen por no interpretar las consignas, desconocer las nociones involucradas o no poder anticipar determinadas transformaciones geométricas.

Es fundamental que también se valore la devolución de lo producido a los estudiantes, inclusive en las evaluaciones, por medio de diferentes tipos de intercambios - presenciales y/o virtuales - no solo para revisar las propias prácticas y retomar la enseñanza de aquellos aspectos de los contenidos cuyos aprendizajes aún no han sido logrados, sino también para promover que los estudiantes analicen

\footnotetext{
${ }^{10}$ Pueden consultarse proyectos similares en Compiano, B., Giarrizzo, A. y Schell, H. (1999). Matemática y su enseñanza. Problemáticas integradoras desde el álgebra, (p. 101-104). Buenos Aires: Edicial y en Giarrizzo (2007).

${ }^{11}$ Los estudiantes de otros terceros y cuartos años también realizaron proyectos similares y expusieron los materiales didácticos en una muestra institucional abierta a la comunidad.
} 
y transformen sus conocimientos. Para cerrar este trabajo, se recupera una cita de una publicación de Sadovsky (2005) que resulta relevante para lo discutido en el presente artículo:

Hay un asunto de confianza como trasfondo del tipo de vínculo que se entabla entre el alumno y el docente a raíz de los intercambios que sostienen: ¿hasta qué punto el docente confía en las posibilidades del alumno de producir ideas?, ¿hasta qué punto las prestigia como parte de la producción relevante de la clase?, ¿hasta qué punto el alumno confía en que el docente no juzgará de modo negativo las marchas y contramarchas propias de la gestación de conocimiento?(...) que el estudiante produzca conocimiento ubica al profesor en un vínculo de intercambio intelectual en el que todas las ideas del alumno -erróneas, provisorias, imprecisas, pertinentes, brillantes- puedan tener un valor para la producción. (p. 15)

\section{§6. Agradecimientos}

A mis exalumnos, docentes y directivos de instituciones educativas de todos los niveles de enseñanza con los que realicé o asesoré la puesta en práctica de experiencias similares. A los colegas y especialistas con los que compartí investigaciones, producciones escritas, eventos educativos y encuentros de capacitación. A los revisores y al Comité Editorial de esta revista por las valiosas orientaciones recibidas durante la producción del artículo.

\section{Bibliografía}

Alsina, C., Burgués, C., y Fortuny, J. M. (1988). Materiales para construir la geometría. Madrid: Editorial Síntesis S.A.

Area, M., Parcerisa, A., y Rodriguez, J. C. (2010). Materiales y recursos didácticos en contextos comunitarios. Barcelona: Editorial Grao.

Bressan, A. M., Bogisic, B., y Crego, K. (2006). Razones para enseñar geometría en la educación básica. Mirar, construir, decir y pensar... Buenos Aires: Ediciones Novedades Educativas.

Coriat, M. (1997). Materiales, Recursos y Actividades: Un panorama. En L. Rico (Ed.), La educación matemática en la enseñanza secundaria (p. 155-178). Barcelona: Horsori.

de Guzmán, M. (2010). El rincón de la pizarra: ensayos de visualización en análisis matemático: elementos básicos del análisis. Madrid: Ediciones Pirámide. 
Duval, R. (2006). Un tema crucial en la educación matemática: la habilidad para cambiar el registro de representación. Gaceta de la Real Sociedad Matemática Española, 9(1), 143-168. Descargado de https: / / gaceta.rsme.es / abrir.php?id=546

Giarrizzo, A. (2007) . ¿Proporcional o no proporcional? Construcciones geométricas con sentido. Novedades Educativas, 18(195), 66-70.

Giarrizzo, A. (2016). Relaciones espaciales y cuerpos geométricos. Resolución de problemas matemáticos en el nivel inicial. ( $\left.N^{\circ} 103\right)$. Buenos Aires: Novedades Educativas. Colección 0 a 5, la educación en los primeros años.

Gimeno Sacristán, J. (2001). Docencia y cultura escolar. Reformas y modelos educativos. Buenos Aires: Lugar Editorial.

González Marí, J. L. (2010). Recursos, Material didáctico y juegos y pasatiempos para Matemáticas en Infantil, Primaria y ESO: consideraciones generales. Descargado de https://bit.1y/3ijonsv

Hernán, F., y Carrillo, E. (1988). Recursos en el aula de matemáticas, (34). Madrid: Editorial Síntesis. Colección Matemáticas: Cultura y Aprendizaje.

Sadovsky, P. (2005). Enseñar Matemática hoy. Miradas, sentidos y desafíos. Buenos Aires: Libros del Zorzal.

Alicia Mirta Giarrizzo

Docente jubilada. Profesora de Matemática y Cosmografía. Licenciada en Educación con orientación en Enseñanza de la Matemática. Capacitadora en talleres, jornadas y eventos educativos para las editoriales Santillana y Novedades Educativas. Asesora de Instituciones Educativas. Escritora de artículos y libros relacionados con la enseñanza y el aprendizaje de la Matemática.

(凶) agiarriz@gmail.com

Recibido: 3 de agosto de 2020.

Aceptado: 28 de junio de 2021.

Publicado en línea: 31 de julio de 2021. 